\title{
Spectrum Opportunity Detection in Cellular Networks
}

\author{
Astha Sharma, Laxman Singh
}

\begin{abstract}
The signal propagation over wireless channels cannot be predicted perfectly due to numerous factors such as fading, channel interference and obstacles. An interference footprint is required to be estimated accurately for evaluation of the spatial spectrum opportunity. It is difficult to determine the spatial spectrum opportunities available in uplink bands of cellular networks due to different location of primary users at different times. In this research work, spatial spectrum opportunity in uplink bands of cellular network is determined using an efficient computational geometry tool for realistic scenario. Our results shows that the performance of umbrella footprints based approach is better than that of conventional circular footprints based approach in terms of false alarm and missed detection probabilities.
\end{abstract}

Keywords: Interference Model; Opportunistic Spectrum Access; Power Control; Spatial spectrum opportunity; Umbrella Diagram.

\section{INTRODUCTION}

Dynamic Spectrum Access (DSA) is considered to be a new spectrum sharing paradigm that gives permission to the unlicensed secondary users (SU's) to access the abundant spectrum holes that are present in licensed bands in an opportunistic manner. An accurate prediction of the spectrum holes available in the primary wireless networks leads to the major problems in the deployment of SU's. These spectrum holes represent the Spectrum Opportunity (SO) which defines the potential opportunities existing in multidimensional regions typically within time, frequency or space [1-2]. In general, SO can be categorized as temporal or spatial [3]. Temporal SO corresponds to the idle time slots, whereas spatial SO corresponds to the geographical area, wherein, secondary user may be located far away from the primary user. Most of the research papers have worked on temporal spectrum opportunities detection [3-7], while limited research is done on spatial spectrum opportunity detection [8-11]. This paper gives emphasis on finding spatial spectrum opportunities available in uplink bands of Cellular Network. According to the ref. [12, 13], both the downlink and uplink bands are the ideal candidates for secondary reuse

Revised Manuscript Received on December 30, 2019.

* Correspondence Author

Astha Sharma, Department of Electronics \& Comm. Engineering, IILM-AHL-College of Engineering \& Technology, Gr. Noida, India.

Laxman Singh", Dept. of Elect. \& Comm. Engineering, Noida Institute of Engineering \& Technology, Gr. Noida, India.

(c) The Authors. Published by Blue Eyes Intelligence Engineering and Sciences Publication (BEIESP). This is an open access article under the CC BY-NC-ND license (http://creativecommons.org/licenses/by-nc-nd/4.0/) for GSM network However, it is not easy to compute the spatial SO in uplink bands due to the varying locations of the primary transmitters. Protocol model, interference range model and physical model are considered as most widely used interference models [14-15]. However, these models are not found suitable for realistic scenarios. As these models simply uses the circular interference ranges for wireless networks and and omni-directional for radio propagation networks.

Kamakaris et al. [16] performed the experiment with the two spatial regions namely, Region of Interference (RoI) for SU receivers and Region of Communication (RoC) for SU transmitters. In this paper, authors illustrated that the antipodal area of cell may be used by secondary network owing to non-utilization of the bandwidth in this region. It happens due to the power control strategies adopted for the network. The SU and PU transmission powers and topology of the network are taken into consideration, while constructing the regions with interference constraints. In general, a spatial region measures the spatial coherence of resources used by primary mainly in case of frequency channels, and CDMA channels. Umbrella diagram algorithm is usually used to determine the spatial regions due to its greater capability to deal with the spatial regions [17]. Computational geometry is used for the design and analysis of an efficient algorithm for geometric related problems [18-20]. The umbrella diagram algorithm is capable of computing highly accurate regions around secondary user's node by partitioning the primary network. The network will be partitioned in such a manner that the secondary user lying in a region will have a larger distance to nodes of the primary user. Hence, an umbrella-based algorithm can be used as an efficient computational tool for analysing the realistic coverage through evaluating the spatial interference footprints. The evaluation of realistic interference footprints could help network designers to (i) design energy-efficient wireless networks, (ii) form deployment strategies for cost-efficient coverage and node scheduling. In this paper, the performance of umbrella-based interference footprints approach is analysed over the conventional circular interference approach. The rest of the paper is arranged as follows. Sections 2 discuss the system model by briefly explaining the concept of Umbrella diagram algorithm. Thereafter, the implementation of umbrella based algorithm for spectrum opportunity detection is given in Section 3. Numerical results are given in Section 4. Finally, the conclusions are summarized in Section 5. 


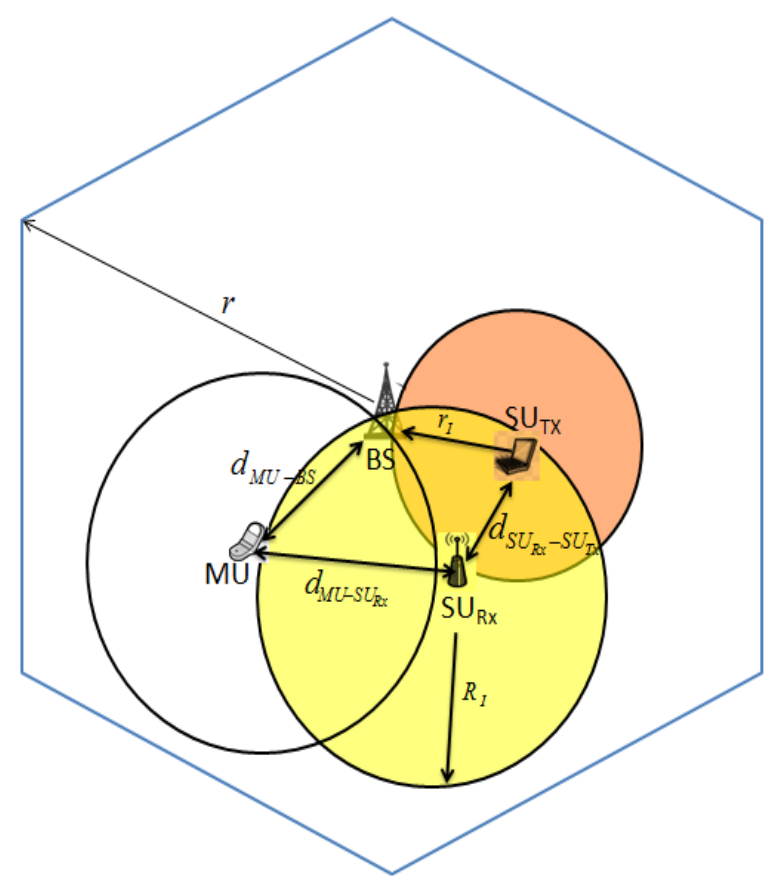

Fig1: System model

\section{SYSTEM MODEL}

Consider the following system model as shown in Figure 1, where SUTx communicates to its intended SURx utilizing the same uplink resources that the mobile user (MU), acting as PUTx is communicating to the base-station (BS) which is PURx within a cell. Throughout the paper we have used PUTx for MU and PURx for BS notations interchangeably. Since MU employ FDD mode for their transmission, hence BS can receive only from a single MU. Similar to [16], it is assumed that PUTx follow power control strategy such that its transmission range is bounded w.r.t. its distance from BS. The circle centred at SURx of radius $R_{I}$ represents the interference range of $\mathrm{PU}$ which is dependent on $\mathrm{PU}$ transmission power and SURx interference tolerance. While, the small circle centred at SUTx of radius $r_{I}$ represents the interference range of SU which depends on SUTx transmission power and the interference constraint which defines the maximum power allowed for SU transmission such that it would be operating below the interference range of the BS [20].

Let $\mathrm{P}_{\mathrm{T}}^{\mathrm{L}}, \mathrm{P}_{\mathrm{R}}^{\mathrm{L}}$ be the transmitted and received power of node $\mathrm{L}$, and let $d(\mathrm{~L}, \mathrm{M})$ represent the distance between node $\mathrm{L}$ and $\mathrm{M}$. Assuming a path-loss gradient $\beta$, the power received by the base-station $B S\left(\mathrm{P}_{\mathrm{R}}^{\mathrm{BS}}\right.$ ) can be expressed as [16]:

$$
\mathrm{P}_{\mathrm{R}}^{\mathrm{BS}}=\frac{\mathrm{P}_{\mathrm{T}}^{\mathrm{MU}}}{\mathrm{d}(\mathrm{MU}, \mathrm{BS})^{\boldsymbol{\beta}}} \Rightarrow \mathrm{P}_{\mathrm{T}}^{\mathrm{MU}}=\mathrm{P}_{\mathrm{R}}^{\mathrm{BS}} \mathrm{d}(\mathrm{MU}, \mathrm{BS})^{\boldsymbol{\beta}}
$$

In order to retrieve the received signal by secondary user's receiver (SUR) with an interference range $\mathrm{R}_{\text {Intf }}$, the condition that should be satisfied is given by

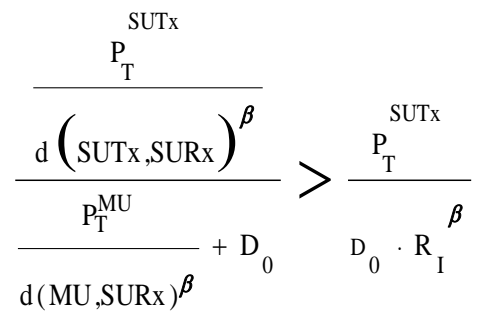

Here SUT represent secondary user's transmitting node. In case, the assumption of the power control strategy is taken into account, the inequality condition given below must be true by the MU in order to interfere with SU :

$$
\mathrm{d}(\text { MU, SURx })^{\beta} \leq \mathrm{d}(\mathrm{MU}, \mathrm{BS})^{\beta}<\mathrm{r}
$$

where $r$ is the cell radius. The strategy adopted here is motivated by the strategy discussed in Ref. [17] where the two spatial regions named $\mathrm{RoI}$ and $\mathrm{RoC}$ are computed using the above equations. To determine the SU location within a cell, RoI locate the area within which transmitting node of primary user will make those resources unavailable to the secondary user. However, on the other side, RoC locate the area within which a secondary user can communicate to the receiver to whom it wants to communicate without interference with any other primary user [16]. During this process of communication, SU transmitter should not increase the Signal-to-Interference ratio (SINR) that is observed at BS for the primary user's transmitting node. Usually, the PUTx's distribution remains unknown across the adjacent BS's, the transmission of SU needs to pose a limit on the coverage range and that should be less than the distance of the closest BS. The two spatial regions $R o I$ and $R o C$ are designed with the help of MATLAB software.In the proposed approach, the circular polygon indicates the interference range of secondary user's node. The circular polygon signifies the range within which secondary user's transmitting node can successfully communicate to secondary user which are located within its allocated range. While for secondary user's receiving node, this circle gives the information about the sensitivity range within which SUR would have the enough sensitivity to detect the presence of transmitting node of any primary user. The main role of Umbrella diagram algorithm is to design \& construct a region that is formed by the perpendicular bisectors (or their extensions) between SUTx or SURx and centres of cells $[16,17]$.

\section{SPECTRUM OPPORTUNITY DETECTION USING UMBRELLA SPATIAL FOOTPRINTS}

\section{A. Performance Metrics for Sspectrum Opportunity Detection}

Spectrum opportunity depends upon the transmitting as well as receiving activities of primary user. Author of Ref. [20] has demonstrated that spectrum opportunity depends upon the three parameters: (i) transmission powers of both primary and secondary users, (ii) the position of these users geographically, and (iii) the interference constraint. 
To measure the detection performance the probabilities of miss detection and false alarm are used as performance metrics:

$$
\begin{aligned}
& P_{f a}=\operatorname{Pr}\left\{{\text { decides } \left.H_{1} \mid H_{0}\right\}}_{\mathrm{P}_{\mathrm{MD}}}=\operatorname{Pr}\left\{{\text { decides } \left.\mathrm{H}_{0} \mid \mathrm{H}_{1}\right\}}^{\text {decila }}\right.\right.
\end{aligned}
$$

The performance of detector is given by the receiver operating characteristic (ROC) curve which gives probability of detection as a function of false alarm probability for varying threshold value. The geographic distribution and traffic pattern of PUs causes uncertainties which lead to three possible sources of detection errors: hidden transmitters, hidden receivers, and exposed transmitters [20].The hidden terminals represents the scenario wherein the primary user is transmitting but is mistakenly considered as a white space. On the other side, the terminal which is not hidden leads to situation where the spectrum is available but is mistakenly sensed as being occupied by the primary user. Therefore, the hidden terminals are considered as source of miss detections while exposed transmitter can be viewed as the source of false alarms.

\section{B. Mathematical Background of SO performance metrics}

The seconadary gets an opportunity when there is no PURx in the proximity of SUTx in that RoC area and no PUTx is available in the RoI area close to SU Rx. Taking these assumptions, into consideration, the $R o C$ is constructed in such a manner so that SUTx can operate below the interference range of base station. Spectrum opportunity probability is assumed as independent to $\mathrm{RoC}$ area and is denoted by $A_{C}$. SURx had the chance to get a spectrum opportunity if no PUTx's are available inside of RoI. Let $\mathrm{A}_{\mathrm{I}}$ denote the RoI area for associated secondary transmitter and let $A$ represents the total cell area then probability of opportunity for the assumed umbrella interference model can be given as:

$$
\operatorname{Pr}\left[H_{0}\right]=1-\frac{\mathrm{A}_{\mathrm{I}}}{\mathrm{A}}
$$

$\mathrm{A}_{\mathrm{I}}$ is dependent on $\mathrm{PU}$ transmission power and interference tolerance of SURx. Once the probability $\operatorname{Pr}\left[H_{0}\right]$ is known, the probability of false alarm and probability of missed detection based on spectrum opportunity definition can be computed as:

$$
\begin{aligned}
P_{f a}= & \operatorname{Pr}\left\{\Lambda\left(A, r_{D}, t x\right) \mid H_{0}\right\} \\
= & 1-\operatorname{Pr}\left\{\overline{\Lambda\left(A, r_{D}, t x\right)} \mid H_{0}\right\} \\
& \text { For umbrella interference model, the logic }
\end{aligned}
$$
condition $\overline{\Lambda\left(A, A_{C}, r x\right)}$ will always be true as for the considered system model and according to assumed power control strategy, base station will never lie within $\mathrm{A}_{\mathrm{C}}$.

Similarly, probability of missed detection can be expressed as :

$$
P_{m d}=\operatorname{Pr}\left\{\overline{\Lambda\left(A, r_{D}, t x\right)} \mid H_{1}\right\}
$$

An outage occurs when the PURx is not able to achieve adequate reception of a signal for which the SNR adjustments by a factor of $d / d_{0}$ needs to be done for both $\mathrm{RoI}$ and RoC as given in [16]. Here, $d$ signifies the distance between BS and SU while $d_{0}$ denotes the reference distance. For avoiding the primary system to go in outage, the signal power of PUTx needs to be increased leading to increased SNR level which further results in increased RoI by a factor of $d / d_{0}$ as the size of the region is dependent on the transmission power of PU. On the other hand, the secondary user needs to limit its transmission for communicating by reducing the size of RoC by a factor of $d_{0} / d$ so that SINR level remains large. Thus, by adjusting the two spatial regions by this factor of $d / d_{0}$, the secondary user communication is still possible by satisfying the interference constraint set by primary system.

\section{RESULT AND DISCUSSION}

We assume a hexagonal layout with radius $r=1$ to get the simulation results. Single primary user's transmitting nodes and SUTX-SURx pair which are uniformly distributed inside the main centre cell are considered to take part in communication. The SURx is assumed to lie within the radius $r_{I}$ of SUTx. The Monte Carlo simulation is run for different locations of PUTx and SUTx, which is denoted by $N$ and $M$ respectively in this paper. In this study, the value of $\mathrm{N}$ and $\mathrm{M}$ are chosen as: $\mathrm{N}=480$ and $\mathrm{M}=480$. In order to successfully detect any activity by primary user within SU detection range, secondary user must have an efficient detection capability.

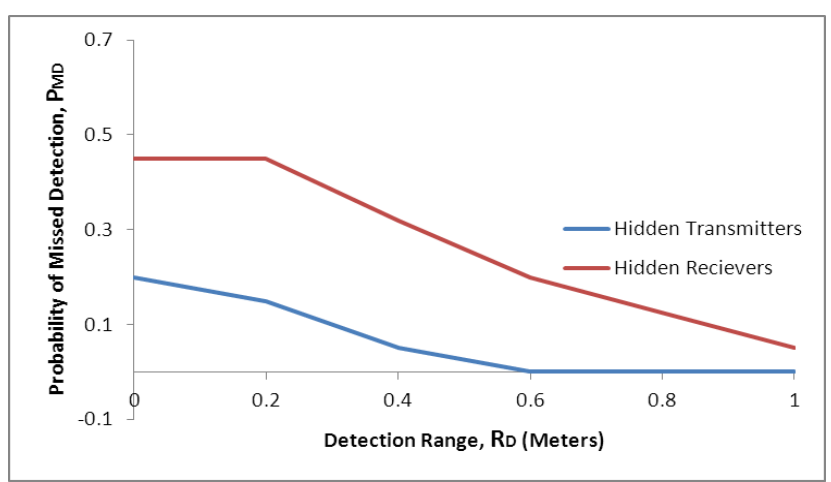

Fig. 2.Plot of Detection range Vs probability of miss detection for both hidden transmitters and hidden receivers

A poor detection sensitivity can increase the missed detection rate for primary users which can cause unacceptable interference to other system from secondary transmissions. The missed detection probability can be used due to occurrence of hidden transmitter and hidden receiver as shown In Fig. 2.It can be observed from the results that the low detection range leads to the high miss detection probability while high detection range leads to low missed detectability probability allowing the user higher mobility for roaming. 


\section{Spectrum Opportunity Detection in Cellular Networks}

In addition to this, the missed detection probability due to presence of hidden receiver occurs for $r_{d}<r_{I}$ whereas this probability is zero for $r_{d} \geq r_{I}$. This implies that detection range is required to be very high in comparison to secondary user's interference range for the successfully detection of the hidden and exposed nodes present within the system. Here, hidden receiver problem does not occur because of the construction of RoC based on the assumed power control strategy where SU constrain its transmission range proportional to its distance from base station centre. Receiver Operating Characteristics (ROC) curve is plotted in Fig. 3. ROC curve is plotted by adjusting the detection range where it can be clearly seen that probability of false alarm increases while probability of detections decreases with an increase in $r_{d}$.

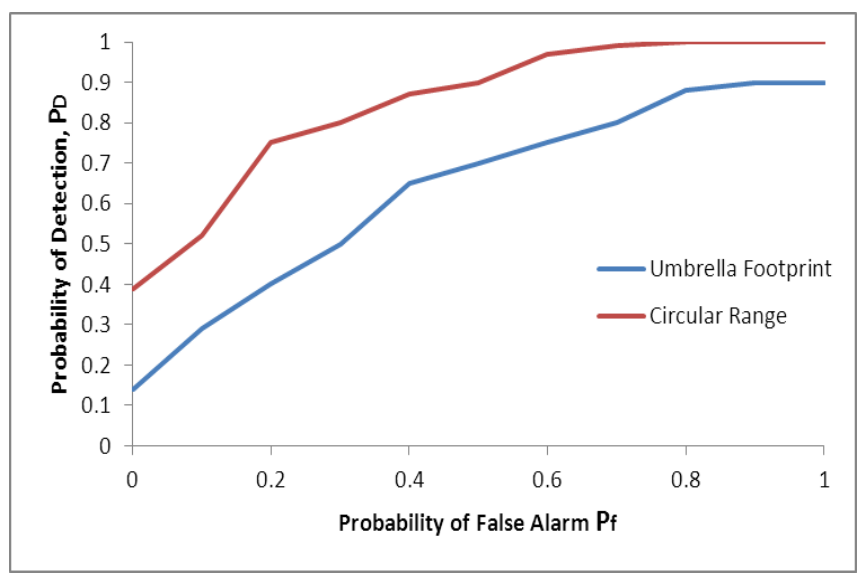

Fig. 3. Receiver operating characteristics for umbrella footprint and circular range methods.

The benefit of evaluating umbrella interference footprint over the circular range is shown by plotting the probability of missed detection, $P_{m d}$ caused due to combined occurrence of both hidden transmitter and receiver for varying range of detection, $r_{d}$ in Fig. 4, where it can be seen that umbrella footprint provides low value of missed detection probability for almost same value of detection range while comparing with circular approach.

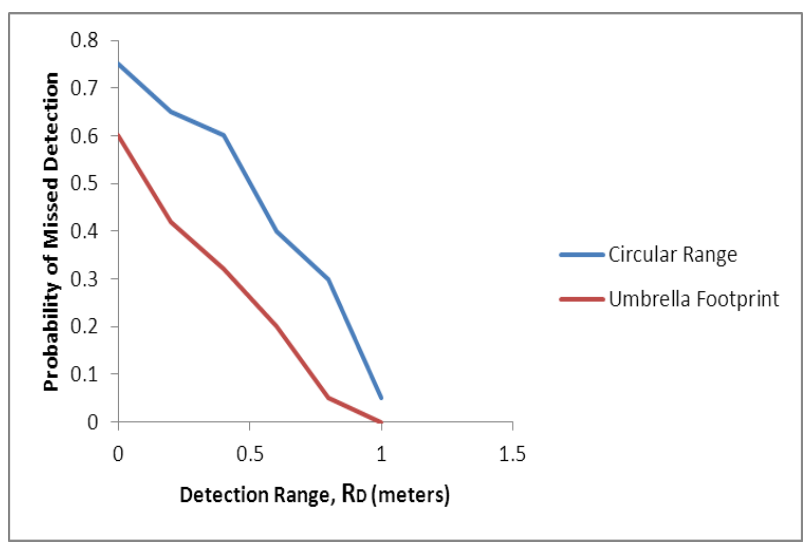

Fig. 4.Plot of Detection range Vs probability of miss detection for both hidden transmitters and hidden receivers

\section{CONCLUSION}

In this paper we investigated the performance of opportunity detector in terms of missed spectrum opportunities and collisions with primary users, when the interference regions for the realistic scenario are computed using umbrella diagram algorithm. Through results, we have shown that umbrella-based interference footprints leads to better utilization of spectrum as compared to circular interference ranges by allowing the SU to maximize its transmission opportunity without sacrificing the desired degree of protection for primary users. Furthermore, the probabilities of missed detection with varying detection ranges were also analysed. In this paper, it is concluded that the detection range needs to be larger than interference range as small detection range may lead to increased probability of missed detection and false alarm. Results reported in this paper may be useful for designing MAC protocols and algorithms for cognitive cellular networks to create and maintain spectrum sharing opportunities.

\section{REFERENCES}

1. Q. Zhao and B. M. Sadler, "A Survey of Dynamic Spectrum Access," IEEE Signal Processing Mag., vol. 24, no. 3, pp. 79-89, May, 2007.

2. J. Ma, G. Zhao and Y. Li. 2008. Soft combination and detection for cooperative spectrum sensing in cognitive radio networks. IEEE Transactions on Wireless Communications,7(11), pp.4502-4507.

3. You Han a, Eylem Ekici , Haris Kremo ,Onur Altintas 2016. Spectrum sharing methods for the coexistence of multiple RF systems: A survey. Ad Hoc Networks. 53, 53-78, 2016.

4. Y.A and N.Kaabouch 2019. A Comprehensive Survey on Spectrum Sensing in Cognitive Radio Networks: Recent Advances, New Challenges, and Future Research Directions. Sensors, 19, 126. Doi:10.3390/s19010126.

5. W.K.ID and H.Yu. Sum Utilization of Spectrum with Spectrum Hand Off and Imperfect Sensing in Interweave Multi Channel Cognitive Radio Network. 10: 1764, 2018. Doi:10.3390/su10061764.

6. R.Umar and A.U.Sheikh.2013. A comparative study of spectrum awareness techniques for cognitive radio oriented wireless networks. Physical Communication, 9, pp. 148-170.

7. Federal Communications Commission, Tech. Rep. 08-260, Nov. 2008. In the Matter of Unlicensed Operation in the TV Broadcast Bands: Second Report and Order and Memorandum Opinion and Order.

8. K.M.Kang, J.C.Park, S.I.Cho, B.J.Jeong, Y.J.Kim,H.J.Lim and G.H. Deployment and coverage of cognitive radio networks in TV white space. Commnication Magazine, IEEE, 50(12), pp.88-94.

9. R.Dhama, K.W.Sowerby, and G.B.Rowe.2011. Capacity of an Ad Hoc Network Sharing Spectrum with a Broadcast Primary System. In Virginia Tech Symp. On Wireless Personal Communication, Blacksburg.

10.D. Tsolkas, P.Nikos and M.Lazaros. Spatial Spectrum Reuse for Opportunistic Spectrum Access in Infrastructure-Based System. Wireless personal communication 69, no.4 (2013):1749-1772.

11. Y. Zhang, L. Bao, M. Welling and S.H. Yang. 2009. Base station localization in search of empty spectrum spaces in cognitive radio networks. In MSN (pp- 94-101).

12. M. Vu, N. Devroye and V. Tarokh. On the primary exclusive region of cognitive networks. IEEE Transaction on Wireless Communication, Vol. 8, pp. 3380-3385, Jul. 2009.

13. Y. Zhao, B. Panigrahi, K. Sohraby and W. Wang. 2013. Interference modeling and analysis in cognitive radio networks. International Journal of Handheld Computing Research (IJHCR), 4(4), pp 1-15.

14. A. Iyer, C. Rosenberg and A. Karnik. 2009. What is the right model for wireless channel interference ? IEEE Transactions on Wireless Communications, 8(5), pp. 2662-2671.

15. T. Kamakaris, , D. Kivanc-Tureli, and U. Tureli, "Interference model for cognitive coexistence in cellular systems," Global Telecommunications Conference, 2007. GLOBECOM'07. IEEE. IEEE, 2007.

16. G. Fan and J. Zhang. A novel geometric diagram and its applications in wireless networks. INFOCOM 2004. Twenty-third AnnualJoint Conference of the IEEE Computer and Communications Societies. Vol. 1. IEEE, 2004. 
17.D.B. Mark, et al. Computational geometry. Springer Berlin Heidelberg, 2000.

18.Q. Zhao. Spectrum Opportunity and Interference Constraint in Opportunistic Spectrum Access. ICASSP (3). 2007.

19.W. Ren, Q. Zhao, and A. Swami. Power control in cognitive radio networks: How to cross a multi-lane highway. IEEE Journal on Selected Areas in Communications, 27.7 (2009): 1283-1296.

20.X. Song, C. Yin, D. Liu and R. Zhang. 2014. Spatial throughput characterization in cognitive radio networks with threshold-based opportunitic spectrum access. Selected Areas in Communications, IEEE Journal on, 32(11), pp. 2190-2204.

\section{AUTHOR PROFILE}

Astha Sharma received her $\mathrm{PhD}$ in Electronics Engineering from Indian Institute of Technology (Indian School of Mines), Dhanbad in 2018. She has received her M.Tech. degree in Electronics and Communication Engineering from Jaypee Institute of Information Technology, Noida in 2011. In 2013, she has worked as Doctoral Exchange Student for 10 months at Department of Electrical, Electronic, and Information Engineering "Guglielmo Marconi", in University of Bologna, Italy under the Erasmus Mundus India4eu II action program. Currently, she is working as Assistant Professor at Department of Electronics and Communication Engineering in IILM-AHL College of Engineering and Technology, Greater Noida. Her research interest includes cognitive radio networks, wireless communication, digital communication, signal processing and machine learning.

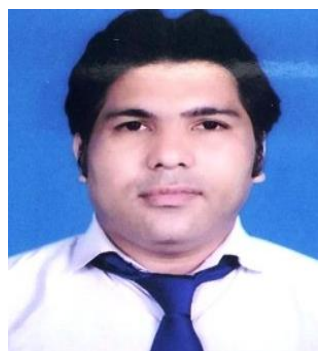

Dr. Laxman Singh obtained his B. Tech in Electronics and Communication Engineering from C.R. State (Govt.) College of Engineering, Murthal, Sonipat (Haryana) and M.Tech in Instrumentation and Control from M.D. University, Haryana, India in 2004 and 2009 respectively. He recieved his $\mathrm{PhD}$ degree from Jamia Millia Islamia (a central Govt. of India University) in 2016. Presently he is working as Associate Professor in the Department of Electronics \& Communication Engineering at Noida Institute of Engineering \& Technology (NIET), Greater Noida. He has total teaching experience of fifteen years. Dr. Laxman Singh has published many research papers in the area of Electronics and Communication Engineering in refereed international /national journals and conferences. His current research interests are in the areas of Wavelet analysis, Artificial Intelligence, Image processing, and Optimization techniques. 